\title{
Otimização de Energia em uma Rede de Satélites LEO em Cenários de Alta Vazão
}

\author{
Renata do Nascimento Mota \\ Macambira \\ Universidade Federal do Amazonas \\ reatamota@gmail.com
}

\author{
Celso Barbosa Carvalho \\ Universidade Federal do Amazonas \\ celsocarvalho75@gmail.com
}

\author{
José Ferreira de Rezende \\ Universidade Federal do Rio de \\ Janeiro \\ jfrezende@gmail.com
}

\begin{abstract}
Low Earth Orbit (LEO) satellites, when exposed to the sun, use solar energy for operation, processing, and communication, and with excess energy they charge their batteries. However, when satellites are in an area with no sunlight, called eclipse areas, they operate using only their battery power. The batteries have limitations on the amount of recharges/discharges, also known as the depth of discharge (DOD) cycle. Therefore, this restricts the useful life of the batteries themselves and also of the satellites.

In this paper, we propose two different efficient routing methods for LEO satellite networks, which optimize traffic in order to reduce the DOD of satellites. We improved the Energy and Capacity Aware Routing (ECARS) metric, existing in the literature, by adding the Energy Routing prUning (ERU)-DOD and Energy Routing penAlty (ERA)-DOD methods. These proposed methods prune and penalize, respectively, the links whose satellites have reached a certain minimum battery charge threshold. With this procedure, we avoid over-discharging the satellites' battery, and thus, the lifetime is extended.

Simulations results show that ERU-DOD and ERA-DOD can increase the satellites' batteries lifetime by more than $54 \%$ and $10 \%$, respectively. Moreover, the average residual energy obtained when comparing our ERU-DOD and ERA-DOD proposals with the ECARS proposal, resulted in gains greater than $113 \%$ and $29 \%$, respectively.
\end{abstract}

\section{KEYWORDS \\ SATÉLITES LEO, ECLIPSE, VIDA ÚTIL DA BATERIA, ROTEAMENTO, ENERGIA}

\section{INTRODUÇÃO}

Atualmente, existe a necessidade de estar conectado o tempo todo e, essa demanda só tende a crescer. No mundo são cerca de 4,1 bilhões de pessoas ligadas à Internet, ou seja, cerca de 59\% da população mundial [1]. No Brasil, cerca de 127 milhões de pessoas tem acesso à Internet, correspondendo à cerca de $60 \%$ da população do país [2], havendo, portanto, muito espaço para crescimento.

Com o aumento da comunicação global, as redes de satélites estão recebendo crescente atenção de empresas e pesquisas. As redes de satélites desempenharão um papel indispensável na $6^{\mathrm{a}}$ geração de comunicação e conectividade, devido à sua grande capacidade de transmissão de dados e cobertura global, permitindo o acesso às mais remotas localidades do globo, como florestas, desertos e oceanos, além de permitir conexão dentro de aviões, o que as torna fundamental para o atendimento à crescente demanda de comunicação de pessoas [3] e sensores nas redes IoT em aplicações como monitoramento ambiental ou previsão de incêndios florestais [4].

Os satélites LEO (Low Earth Orbit - Órbita Terrestre Baixa) cobrem toda a superfície terrestre através da cooperação de um conjunto de satélites, denominado constelação. Neste artigo, foi considerada a constelação Iridium, composta por 66 satélites LEO distribuídos em seis planos orbitais, cada plano com onze satélites e localizados a $780 \mathrm{~km}$ acima do solo [5], fornecendo principalmente serviço de voz e de dados com baixa taxa.

Os satélites LEO ficam em constante movimento ao redor da Terra, o que os faz entrarem em áreas de eclipse, ou seja, áreas sem incidência da luz solar. Quando expostos ao sol, os satélites LEO utilizam a energia solar para operação, processamento e comunicação, e com a energia excedente carregam suas baterias.

Entretanto, em áreas de eclipse operam utilizando apenas a energia de suas baterias. As baterias possuem limitação na quantidade de recargas/descargas, também conhecido como ciclo de profundidade de descarga (DOD - Deep of Discharge), de forma que isto restringe a vida útil das próprias baterias e consequentemente dos satélites.

Neste artigo propomos um método para balanceamento de carga no roteamento de pacotes nos satélites de uma constelação LEO, limitando o nível mínimo da bateria, a fim de aumentar a vida útil das baterias dos satélites. Dessa forma, apresentamos dois métodos denominados ERU-DOD (Energy Routing prUning - Depth of Discharge) e ERA-DOD (Energy Routing penAlty Depth of Discharge) que adaptam a métrica de roteamento proposta por [6]. Aplicamos o algoritmo de caminho com menor custo, Dijkstra, em conjunto com os métodos de roteamento ERUDOD e ERA-DOD, a fim de encontrar os caminhos mais vantajosos em termos de energia.

O ERU-DOD consiste em atribuir o valor infinito de métrica para os enlaces que não atenderam ao limiar mínimo predefinido para a energia residual das baterias dos satélites, isso é conhecido como poda. O ERA-DOD consiste em atribuir um peso maior para os enlaces que não atenderam a esse limiar mínimo, ou seja, uma penalização. 
Os resultados das simulações mostram que ERU-DOD e ERADOD aumentaram a vida útil das baterias dos satélites em até 54\% e $10 \%$, respectivamente, nos cenários investigados. Além disso, a energia residual média obtida quando comparamos nossas propostas ERU-DOD e ERA-DOD com a proposta ECARS [6], resultaram em ganhos maiores que $113 \%$ e $29 \%$, respectivamente.

O restante deste trabalho é organizado como segue. Na Seção 2 apresenta-se trabalhos relacionados. A Seção 3 apresenta o modelo de roteamento. A Seção 4 apresenta os métodos propostos. Na Seção 5 são mostrados o ambiente da simulação, os resultados experimentais e análise dos resultados. Finalmente, na Seção 6 as conclusões e trabalhos futuros serão apresentados.

\section{TRABALHOS RELACIONADOS}

No trabalho realizado por [7], os autores propuseram as heurísticas BASIC (Basic Enlace Pruning) e SNAP (poSitioN Aware Pruning) para solucionar o problema de minimização do consumo total de energia dos enlaces desativados (podados) em uma rede de satélites LEO. O BASIC é baseado na observação dos enlaces que não estão no caminho mais curto e dificilmente haverá tráfego e, portanto, poderá ser desligado com segurança. $\mathrm{O}$ SNAP remove os enlaces que estão em áreas de eclipse e áreas com pouco tráfego. Esses autores obtiveram $6 \%$ e $12 \%$ de redução da profundidade de descarga, respectivamente, para o BASIC e o SNAP, com limite máximo da utilização do enlace de até $50 \%$, quando comparado com o resultado da topologia sem poda. Entretanto, os autores não limitaram o nível mínimo da bateria dos satélites e nem calcularam a vida útil do satélite.

No que tange o cálculo do ciclo de vida da bateria, em [8], os autores propuseram três algoritmos GreenSR-B, GreenSR-A e GreenSR, com o objetivo de minimizar o custo total de uma rede de satélites em um longo período, controlando o tráfego de rede e colocando os roteadores subutilizados em modo de hibernação, de modo a economizar energia e prolongar a vida útil das células das baterias e, consequentemente, o tempo de vida do satélite. Os autores obtiveram aumento médio do ciclo de vida das baterias dos satélites em 49,97\%; 7,23\% e 41,24\% para os algoritmos GreenSR-A, GreenSR-B e GreenSR, respectivamente. Novamente, os autores observaram apenas o enlace, e não limitaram o nível mínimo da bateria dos satélites, com isso o satélite pode descarregar por completo.

Em [9], os autores utilizaram Q-Lerning para o problema de alocação de energia na comunicação satélite-terra. O objetivo dos autores foi prolongar a vida útil das baterias, controlando a potência de transmissão para o processamento de tráfego dos satélites no mesmo plano orbital. Como resultado, os autores obtiveram um aumento no tempo vida útil das baterias dos satélites LEO em torno de $45 \%$ em média para satélites no mesmo plano orbital. Apesar dos autores controlarem e equalizarem o tráfego, eles não observam a constelação como um todo.

Por fim, o trabalho desenvolvido por [6] também não limita o nível mínimo da bateria dos satélites. Os autores propuseram uma nova métrica denominada ECARS (Energy and Capacicity Aware
Routing - Roteamento Consciente de Energia e Capacidade) com o objetivo de maximizar a demanda de tráfego atendida, minimizando o consumo energético das baterias dos satélites que estão na área de eclipse. Os autores obtiveram 14\% de aumento da demanda atendida. Contudo, os autores não empregaram em sua proposta os limiares para as baterias dos satélites, o que pode acarretar em descarregamento das baterias.

Os trabalhos apresentados acima não aplicaram limiar de carga mínima na bateria dos satélites, fazendo com que a bateria possa descarregar por completo, dessa forma, o satélite fica inutilizado e consequentemente diminui drasticamente sua vida útil. Os métodos propostos neste artigo, ERU-DOD e ERA-DOD, apresentam limiar para as baterias dos satélites, a fim de que as mesmas não descarreguem por completo, e com isso aumentam a vida útil do satélite.

\section{MODELO DE ROTEAMENTO EM REDES DE SATÉLITES LEO}

\subsection{Modelagem do Tráfego}

\section{A. Mapeamento das Zonas Geográficas}

A distribuição de tráfego com os níveis de densidade de usuários por região é mostrada na Figura 1 [10]. Nessa figura, observam-se as regiões da América do Norte, Europa, Ásia, América do Sul, África e Oceania.

Cada uma dessas regiões é limitada por retângulos com bordas destacadas em vermelho. Os quadrados representam as zonas em que o Globo foi dividido, 12 × $24=288$ zonas, com a dimensão da área de cada zona sendo de $15^{\circ}$. Os números rotulados nessas zonas indicam o nível de densidade de usuários $\left(u_{j}\right)$ e o total de zonas consideradas foi igual a 120 .

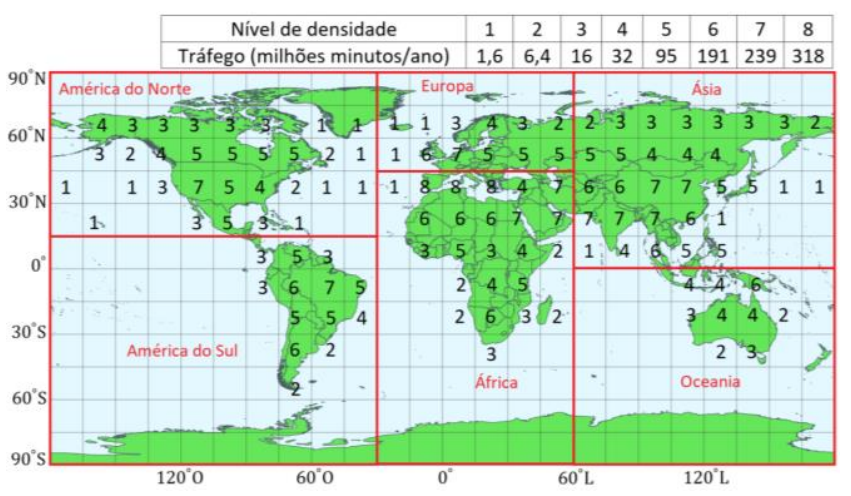

Figura 1 - Divisão da zona terrestre e níveis de densidade de usuário.

\section{B. Número de terminais}


A Tabela 1 mostra o número de terminais de Internet para cada continente, atualizados até junho de 2020 e extraídos do site Miniwatts [11].

Conforme [11], na região da América do Norte estão os seguintes países: Estados Unidos, Canadá, México, Cuba, Porto Rico, Bermuda, Groenlândia e St. Pierre \& Miquelon. Os demais países da América Central e Caribe estão na região de influência da América do Sul.

Tabela 1 - Valores para número de terminais e porcentagem por região [11].

\begin{tabular}{c|c|c}
\hline Região & Número de terminais & $\begin{array}{c}\text { Porcentagem } \\
\text { (\%) }\end{array}$ \\
\hline América do Norte & 436.745 .577 & $7,48 \%$ \\
\hline Europa & 727.848 .547 & $15,63 \%$ \\
\hline Ásia & 2.525 .033 .874 & $54,23 \%$ \\
\hline América do Sul & 371.127 .426 & $7,97 \%$ \\
\hline África & 566.138 .772 & $12,16 \%$ \\
\hline Oceania/Austrália & 28.917 .600 & $0,62 \%$ \\
\hline
\end{tabular}

O número de terminais é utilizado para calcular o nível de densidade de terminais para as diferentes zonas terrestres mostradas na Figura 1. A Equação (1) mostra como é calculada essa densidade [12].

$$
h_{j}=\frac{u_{j} * N_{h}(k)}{\sum_{i} u(i)}
$$

Onde $h_{j}$ é o nível de densidade de terminais da zona $j$, $u_{j}$ é o nível de densidade de usuários da zona $j$, conforme mostrado na Figura 1. $N_{h}(k)$ é o número de terminais do continente $k$ (Tabela 1) e $\sum_{i} u(i)$ é a soma do nível de densidade dos usuários das zonas do continente $\mathrm{k}$.

\section{Matriz de tráfego}

A matriz de tráfego é obtida através do nível de densidade do usuário, nível de densidade de terminais e a distância entre os satélites $i$ e $j$, conforme a Equação (2) [12].

$$
T^{i j}=\frac{\left(u_{i} * h_{j}\right)^{\alpha}}{(d(i, j))^{\beta}}
$$

Onde $\mathrm{T}^{\mathrm{ij}}$ é a matriz de tráfego entre as zonas de origem $i$ até a zona de destino $j, \mathrm{u}_{\mathrm{i}}$ é o nível de densidade de usuários, $h_{i}$ é o nível de densidade de terminais, conforme visto na Equação (1), $d(i, j)$ é a distância entre os satélites $\mathrm{i}, \mathrm{j}$. As constantes $\alpha$ e $\beta$ são definidas como 0,5 e 1,5 , respectivamente [12].

A Tabela 2 ilustra o percentual do fluxo de tráfego entre os continentes, considerando a matriz de tráfego obtida a partir da Equação (2). Em cada linha ou coluna da Tabela 2, a soma dos percentuais de tráfego de cada região (ex. América do Norte) resulta no valor $100 \%$.
Tabela 2 - Valores para número de terminais e porcentagem por região [11].

\begin{tabular}{l|l|l|l|l|l|l}
\hline & \multicolumn{5}{|c}{ Destino } \\
\hline Origem & $\begin{array}{l}\text { América } \\
\text { do } \\
\text { Norte }\end{array}$ & Europa & Ásia & $\begin{array}{l}\text { América } \\
\text { do Sul }\end{array}$ & África & $\begin{array}{l}\text { Oceania } \\
\text { Austrália }\end{array}$ \\
\hline $\begin{array}{l}\text { América } \\
\text { do Norte }\end{array}$ & $\begin{array}{l}54,50 \\
\%\end{array}$ & $\begin{array}{l}11,64 \\
\%\end{array}$ & $\begin{array}{l}20,47 \\
\%\end{array}$ & $7,12 \%$ & $5,60 \%$ & $0,67 \%$ \\
\hline Europa & $7,76 \%$ & $\begin{array}{l}42,75 \\
\%\end{array}$ & $\begin{array}{l}29,60 \\
\%\end{array}$ & $3,07 \%$ & $\begin{array}{l}16,35 \\
\%\end{array}$ & $0,47 \%$ \\
\hline Ásia & $5,28 \%$ & $\begin{array}{l}11,46 \\
\%\end{array}$ & $\begin{array}{l}73,10 \\
\%\end{array}$ & $1,54 \%$ & $7,17 \%$ & $1,45 \%$ \\
\hline $\begin{array}{l}\text { América } \\
\text { do Sul }\end{array}$ & $\begin{array}{l}15,84 \\
0\end{array}$ & $\begin{array}{l}10,24 \\
\%\end{array}$ & $\begin{array}{l}13,26 \\
\%\end{array}$ & $\begin{array}{l}48,87 \\
\%\end{array}$ & $\begin{array}{l}11,02 \\
\%\end{array}$ & $0,78 \%$ \\
\hline África & $5,74 \%$ & $\begin{array}{l}25,14 \\
\%\end{array}$ & $\begin{array}{l}28,47 \\
\%\end{array}$ & $5,07 \%$ & $\begin{array}{l}34,80 \\
\%\end{array}$ & $0,77 \%$ \\
\hline $\begin{array}{l}\text { Oceania } \\
\text { Austrália }\end{array}$ & $7,17 \%$ & $7,63 \%$ & $\begin{array}{l}60,16 \\
\%\end{array}$ & $3,75 \%$ & $8,09 \%$ & $13,21 \%$ \\
\hline
\end{tabular}

\subsection{Cálculo do Ciclo de Vida da Bateria}

O ciclo de vida representa a quantidade de ciclos completos de descarga/recarga possíveis para uma bateria até que a mesma se torne inoperante. No geral, as baterias dos satélites de íon-lítio, utilizadas nos satélites LEO, possuem um total de 60.000 ciclos de vida [8].

A quantidade de ciclos de vida consumida entre o instante $t_{1} \mathrm{e}$ $t_{2}$ é dada na Equação (3):

$$
L_{t_{1} t_{2}}=\left\{\begin{array}{c}
0, \text { se } D\left(t_{1}\right) \geq D\left(t_{2}\right) \\
\int_{D\left(t_{1}\right)}^{D\left(t_{2}\right)} f(D) d D, \text { caso contrário }
\end{array}\right.
$$

Em que $L_{t_{1} t_{2}}$ é a quantidade de ciclos de vida consumidos entre os instantes $t_{1}$ e $t_{2}$. $D\left(t_{1}\right)$ é a profundidade de descarga da bateria no instante $t_{1} . D\left(t_{2}\right)$ é a profundidade de descarga da bateria no instante $t_{2} . f(D)$ é a taxa de consumo dos ciclos de vida quando o DOD é igual a uma descarga $\mathrm{D}$ qualquer, definida como $f(D)=10^{A(D-1)}(1+A * \ln 10 * D)$. A é uma constante que depende das especificações da bateria, neste trabalho, definida como 0,8 .

A profundidade de descarga da bateria (DOD) é dada pela Equação (4) [9].

$$
D O D(t)=\frac{C_{B}^{\max }-C_{B}(t)}{C_{B}^{\max }}
$$

Em que DOD $(\mathrm{t})$ é a profundidade de descarga da bateria de um satélite no instante $t$, variando de 0 a $1 . C_{B}^{\max }$ é capacidade máxima da bateria do satélite. $C_{B}(t)$ é a energia residual armazenada na bateria no instante $t$. 


\section{METODOLOGIA}

Os autores de [6] propuseram uma nova métrica denominada ECARS (Energy and Capacicity Aware Routing - Roteamento Consciente de Energia e Capacidade), Equação (5), com o objetivo de maximizar a demanda de tráfego atendida, minimizando o consumo energético das baterias dos satélites LEO, da constelação Iridium em áreas de eclipse.

$$
E C A R_{S}=w_{1} \frac{T_{k, l}(t)-T_{\min }}{T_{\max }-T_{\min }}+w_{2} \frac{D_{k, l}(t)-D_{\min }}{D_{\max }-D_{\min }}+w_{3} \frac{P_{k, l}(t)-P_{\min }}{P_{\max }-P_{\min }}
$$

Onde, $w_{1}$ é o peso atribuído ao parâmetro $T_{k, l}(t)$, sendo este o tempo de propagação no enlace entre os satélites $k, l$ no instante $t$.

A variável $w_{2}$ atribui peso ao parâmetro $D_{k, l} \cdot D_{k, l}(t)$ é a quantidade que depende do nível de bateria dos satélites $\mathrm{k}$ e 1 no instante $\mathrm{t}, D_{k, l}(t)=\frac{e_{k}}{B_{k}(t)}+\frac{e_{l}}{B_{l}(t)}, e_{k}$ é igual a 1 se o satélite $k$ está em eclipse e 0 caso contrário, $e_{l}$ é igual a 1 se o satélite 1 está em eclipse e 0 caso contrário, $B_{k}(t)$ é a energia residual (em joules) do satélite $k$ no instante $t, B_{l}(t)$ é a energia residual (em joules) do satélite $l$ no instante $t, B_{\max }$ é a energia máxima da bateria, estipulada em 117.000 joules (J).

A variável $w_{3}$ é o peso que pondera o parâmetro $P_{k, l} \cdot P_{k, l}(t)$ é a quantidade que depende da capacidade do enlace $C_{k, l}(t)$ entre os satélites $k$ e $l$ em um determinado instante de tempo $t . P_{k, l}(t)=$ $1-\frac{C_{k, l}(t)}{C_{i s l}}$, onde $C_{k, l}(t)$ é a capacidade (em Mbps) do enlace entre o satélite $k$ e $l$ no instante $t$. $C_{i s l}$ é a capacidade total (em Mbps) do enlace.

O presente artigo adapta a métrica ECARS, através de dois métodos, descritos nas Seções 4.1 e 4.2 seguintes, os quais realizam o balanceamento de carga no roteamento de pacotes nos satélites de uma constelação LEO, limitando o nível mínimo das baterias dos satélites.

\subsection{Método ERU-DOD}

O método ERU-DOD (Energy Routing prUning - Depth Of Discharge) atribui o valor infinito de métrica para os enlaces que não atendem a um limiar mínimo $\left(L_{B}\right)$ de energia residual das baterias dos satélites. O limiar mínimo de energia das baterias dos satélites $\left(L_{B}\right)$ foi simulado e avaliado empiricamente com valores iguais a 0 e 0,5 .

$$
D_{k, l}(t)=\left\{\begin{array}{c}
\left(\frac{e_{k}}{B_{k}(t)}+\frac{e_{l}}{B_{l}(t)}\right), \text { se } \frac{B_{k}(t)}{B_{\max }} \geq L_{B} \text { e } \frac{B_{l}(t)}{B_{\max }} \geq L_{B} \\
\infty, \text { caso contrário; }
\end{array}\right.
$$

A restrição da Equação (6) especifica a carga residual da bateria. Essa restrição é aplicada na Equação (5). Caso não seja atendido o limiar mínimo de bateria, é atribuído o valor infinito ao enlace $(k, 1)$, desabilitando o mesmo. Este procedimento é conhecido como poda.

Nesse modelo, é dado uma atenção maior ao segundo termo da Equação (5), $w_{2} \frac{D_{k, 1}(t)-D_{\min }}{D_{\max }-D_{\min }}$, onde é analisado o nível da bateria dos satélites e aplicado um limiar mínimo de carga e evitando o descarregamento total da bateria do satélite.

Com isso, verifica-se $\frac{B_{k}(t)}{B_{\max }} \geq L_{B}$ e $\frac{B_{1}(t)}{B_{\max }} \geq L_{B}$, ou seja, o limiar é a porcentagem mínima de carga das baterias dos satélites estabelecida como aceitável para que o enlace entre os satélites $k$ e $l$ seja utilizado. Caso o nível da bateria de um dos satélites $k, l$ esteja fora desse limiar é atribuído o valor de infinito para o segundo termo da Equação (5). Logo, o valor da métrica será infinito, e com isso, o enlace é automaticamente desabilitado.

Nesse artigo, os valores de limiar mínimo da bateria $\left(L_{B}\right)$ usados foram de 0 e 0,5 , ou seja, analisando um satélite $\mathrm{k}$ qualquer, a primeira coisa na restrição é checar a razão entre o nível de bateria do satélite $\mathrm{k}$ e a energia máxima da bateria fornecida pelo sistema, dado pela Equação (6), $\frac{B_{k}(t)}{B_{\max }} \geq L_{B}$. Por exemplo, se o satélite k já consumiu $80 \%$ de sua carga de bateria, ele tem 23,4 Kjoules de energia restante, visto que a carga máxima da bateria do satélite é de 117 Kjoules.

Dado o exemplo acima, pela restrição - dada na Equação (6) $\frac{B_{k}(t)}{B_{\max }}=\frac{23,4}{117}=0,2$. Esta razão deve ser maior ou igual ao limiar $\left(\mathrm{L}_{\mathrm{B}}\right)$ predefinido na simulação. No exemplo, se $\mathrm{L}_{\mathrm{B}}$ fosse igual a 0,3 ou 0,5 , todos os enlaces iriam para infinito, visto que a razão resultou em valor menor, igual a 0,2 .

Resumidamente, se o limiar mínimo da bateria $L_{B}$ for $0,2,0,3$ ou 0,5 a métrica permite utilizar os satélites com pelo menos 20 , 30 ou $50 \%$ de carga da bateria restante.

Ao estabelecer um nível mínimo de energia para as baterias dos satélites, evita-se o descarregamento total dessas baterias e, consequentemente, há um aumento considerável de vida útil das baterias, através da redução do DOD no decorrer do tempo.

\subsection{Método ERA-DOD}

O método ERA-DOD (Energy Routing penAlty - Depth Of Discharge) consistiu em atribuir um peso maior para os enlaces que não atendem a um limiar mínimo $\left(L_{B}\right)$ de energia residual das baterias dos satélites, através do aumento do valor do segundo termo da Equação (5).

$$
\begin{aligned}
& {\left[\begin{array}{l}
w_{1} \\
w_{2} \\
w_{3}
\end{array}\right]=\left\{\begin{array}{c}
{\left[\begin{array}{ccc}
0,35 & 0,35 & 0,30
\end{array}\right]^{T}, \text { se } \frac{B_{k}(t)}{B_{\max }} \geq L_{B} \text { e } \frac{B_{l}(t)}{B_{\max }} \geq L_{B}} \\
{\left[\begin{array}{lll}
w_{1}^{T} & w_{2}^{D} & w_{3}^{P}
\end{array}\right]^{T}, \text { caso contrário; }}
\end{array}\right.} \\
& w_{2}^{D}>w_{1}^{T} \text { e } w_{2}^{D}>w_{3}^{P}
\end{aligned}
$$

Onde $w_{1}^{\mathrm{T}}, \mathrm{w}_{2}^{\mathrm{D}}, \mathrm{w}_{3}^{\mathrm{P}}$ são os pesos ou importâncias relativas para os parâmetros tempo de propagação, carga residual da bateria e capacidade do enlace para os satélites dos enlaces que não 
atendem ao limiar mínimo de bateria $\mathrm{L}_{\mathrm{B}}$. A soma dos pesos deve ser igual a 1 .

A restrição da Equação (7) especifica em sua condicional da variável $\mathrm{L}_{\mathrm{B}}$, um valor mínimo de percentual de carga das baterias dos satélites de um enlace $k, l$. Caso este o limiar $\mathrm{L}_{\mathrm{B}}$, não seja atendido, é atribuído um valor maior ao peso $\mathrm{w}_{2}$, conforme mostrado na Equação (8). Essa restrição é referente ao parâmetro $\mathrm{D}_{\mathrm{k}, \mathrm{l}}(\mathrm{t})$ da Equação (5). Este procedimento resulta em aumento do valor do parâmetro e consequente penalização na utilização do enlace.

No método ERA-DOD é dada atenção maior ao segundo termo da Equação (5), $w_{2} \frac{D_{k, 1}(t)-D_{\min }}{D_{\max }-D_{\min }}$, onde é analisado o nível da bateria dos satélites da rede e aplicado um limiar mínimo de carga para a mesma, a fim de inibir o descarregamento total das baterias dos satélites.

Com isso, verificam-se as condições $\frac{B_{k}(t)}{B_{\max }} \geq L_{B}$ e $\frac{B_{1}(t)}{B_{\max }} \geq L_{B}$, ou seja, se a porcentagem de carga das baterias dos satélites de um enlace $k, l$ atende o limiar mínimo $\mathrm{L}_{\mathrm{B}}$. Caso o nível da bateria dos satélites $l, k$ seja inferior a este limiar, é atribuída uma penalização ao segundo termo da Equação (5), ou seja, um peso maior para aquele parâmetro. A restrição, mostrada na Equação (7) especifica a penalização ao segundo termo da Equação (5), ou seja, o peso atribuído ao parâmetro $D_{k, l}(t)$, é maior que os demais pesos, com $\mathrm{w}_{2}^{\mathrm{D}}>\mathrm{w}_{1}^{\mathrm{T}}$ e $\mathrm{w}_{2}^{\mathrm{D}}>\mathrm{w}_{3}^{\mathrm{P}}$, a fim de tornar desvantajosa a utilização de um enlace $k, l$.

Os valores dos pesos $\mathrm{w}_{1}^{\mathrm{T}}, \mathrm{w}_{2}^{\mathrm{D}}$ e $\mathrm{w}_{3}^{\mathrm{P}}$ foram escolhidos empiricamente por meio de experimentos e simulações, conforme Tabela 3 .

\section{AMBIENTE E RESULTADOS EXPERIMENTAIS}

Esta seção apresenta os parâmetros, cenário e ambiente de simulação utilizados neste artigo para avaliar o desempenho dos métodos propostos.

\subsection{Parâmetros e Ambiente da Simulação}

Na Tabela 3 apresentamos os parâmetros e seus valores correspondentes utilizados neste artigo.

As simulações foram realizadas no simulador para redes de satélites LEO, desenvolvido por [6] e implementado em software MATLAB.

No simulador, adaptado neste artigo, é realizada a geração de demandas de tráfego em redes de satélites LEO, a partir dos parâmetros definidos na Tabela 3 . O que permite a simulação em tempo real da constelação Iridium, com todos os seus satélites em suas posições dinâmicas atuais (latitude e longitude) em suas respectivas órbitas.

Utilizou-se, nesta pesquisa, o MATLAB R2017b com um computador DELL Intel® Xeon ${ }^{\circledR}$ com 4 GB de RAM, processador de 1,80-2,40 GHz e Windows 10.
Foram realizadas mais de 100 simulações, com duração de aproximadamente $2 \mathrm{hr}$ cada, tendo gerado quase $150 \mathrm{~Gb}$ de arquivos. Foi simulado a operação da constelação Iridium para 1 dia, equivalendo a um tempo de 90.000 segundos no simulador.

Tabela 3 - Parâmetros e valores utilizados para simulação deste artigo.

\begin{tabular}{|c|c|}
\hline Parâmetro & Valor \\
\hline $\begin{array}{c}\text { Capacidade máxima da } \\
\text { bateria do satélite }\left(B_{\max }\right)\end{array}$ & 117 KJoule $=117.000 \mathrm{~J}$ \\
\hline $\begin{array}{l}\text { Potência de transmissão } \\
\left(P_{t}\right)\end{array}$ & $P_{t}=7 \mathrm{watt}=7 \mathrm{~J} / \mathrm{s}$ \\
\hline Potência de recepção $\left(\mathrm{P}_{\mathrm{r}}\right)$ & $P_{r}=3 \mathrm{watt}=3 \mathrm{~J} / \mathrm{s}$ \\
\hline $\begin{array}{c}\text { Potência de operação } \\
\text { nominal }\left(P_{o}\right)\end{array}$ & $P_{o}=4 w a t t=4 \mathrm{~J} / \mathrm{s}$ \\
\hline Potência de captação $\left(P_{c}\right)$ & $P_{c}=20 \mathrm{watt}=20 \mathrm{~J} / \mathrm{s}$ \\
\hline $\begin{array}{l}\text { Tempo de intervalo de } \\
\text { simulação (snapshot) }\end{array}$ & $100 s$ \\
\hline $\begin{array}{c}\text { Período orbital para o } \\
\text { sistema Iridium }\end{array}$ & $100 \mathrm{~min}$ \\
\hline $\begin{array}{l}\text { Tempo total de simulação } \\
\text { (número de voltas) }\end{array}$ & $90.000 s$ (15 voltas) \\
\hline ISL & $10 \mathrm{Mbps}$ \\
\hline CBR & $1 \mathrm{Mbps}$ \\
\hline $\begin{array}{l}\text { Número de terminais } \\
\text { (fontes) }\end{array}$ & $100,200,400,800,1.000$ \\
\hline $\begin{array}{l}\text { Limiar de carga na bateria } \\
\qquad\left(L_{B}\right)\end{array}$ & 0 e 0,5 \\
\hline Pesos ERU-DOD & {$\left[w_{1}=0,35 ; w_{2}=0,35 ; w_{3}=0,30\right]$} \\
\hline Pesos ERA-DOD & $\begin{array}{c}{\left[w_{1}=0,35 ; w_{2}=0,35 ; w_{3}=0,30\right]} \\
{\left[\mathrm{w}_{1}^{\mathrm{T}}=0,15 ; \mathrm{w}_{2}^{\mathrm{D}}=0,70 ; \mathrm{w}_{3}^{\mathrm{P}}=0,15\right]}\end{array}$ \\
\hline
\end{tabular}

Nas seções seguintes, serão apresentados os gráficos com os melhores resultados, após as simulações com os parâmetros mostrados na Tabela 3 .

\subsection{Energia Residual da Bateria}

Na Figura 2 é mostrada a porcentagem de energia média residual da bateria dos satélites obtida pela utilização dos métodos ERUDOD e ERA-DOD e pela métrica ECARS.

A energia residual é a capacidade máxima da bateria do satélite $\left(B_{\max }\right)$ menos a energia gasta - onde a energia gasta é a soma das energias de transmissão, recepção e operação nominal mais a energia captada.

A energia residual média é calculada pela soma da energia residual de cada um dos satélites dividido pela quantidade total de satélites. 


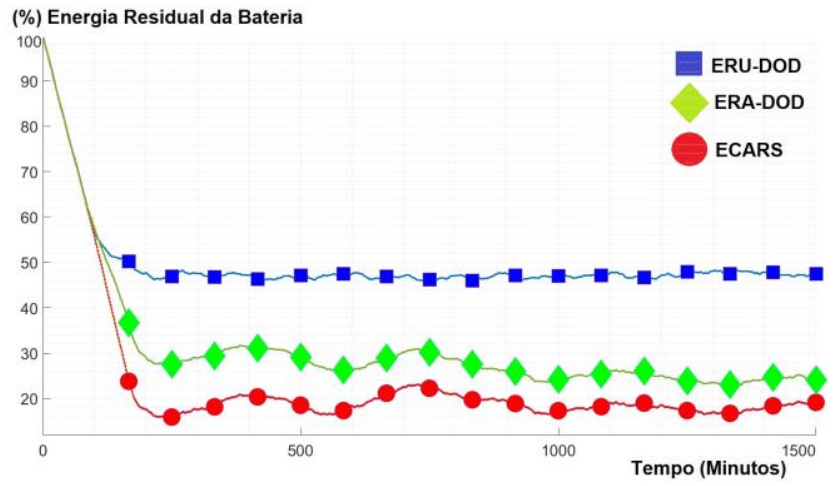

Figura 2 - Média da Energia Residual dos Satélites.

Na métrica ECARS, como não existe limiar mínimo $\left(L_{B}\right)$, a bateria pode descarregar por completo. Dessa forma, a métrica ECARS gera valores de porcentagem de energia residual média para os satélites menor que $20 \%$.

Ao visualizar a Figura 2, nota-se na curva azul (método ERUDOD) que com a utilização do limiar mínimo $\left(L_{B}\right)$ no nível de bateria de 0,5 nos satélites, a média da energia residual dos satélites fica em $50 \%$.

O método ERU-DOD apresenta um ganho de até $113 \%$ em relação ao ECARS. O método ERA-DOD obteve um valor em torno de $29 \%$ maior do que a métrica ECARS.

\subsection{Ciclo de Vida da Bateria}

Na Figura 3 é mostrada a média do tempo de vida útil das baterias dos satélites, obtidas para cada um dos métodos ERU-DOD e ERA-DOD e para a métrica ECARS.

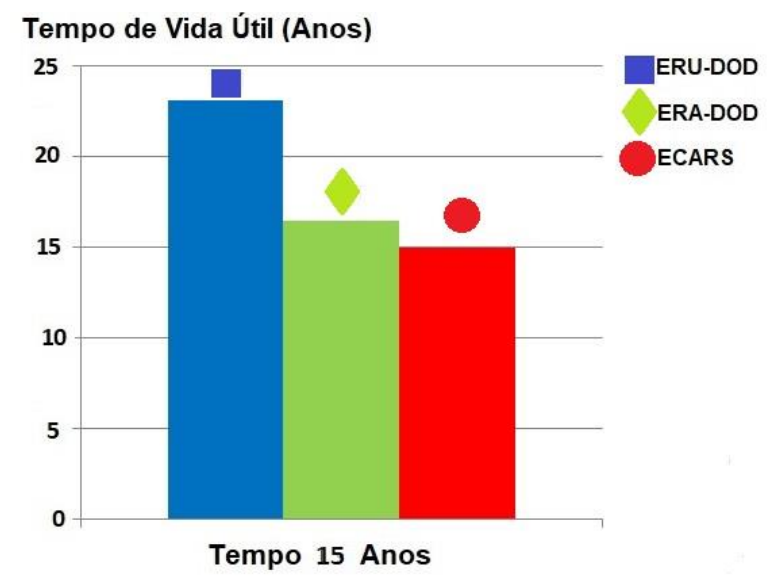

Figura 3 - Tempo de Vida Útil da Bateria dos Satélites.

Quando se aplica um limiar mínimo $\left(L_{B}\right)$ no nível de bateria de 0,5 nos satélites, não se utiliza os satélites com menos de $50 \%$ de energia residual, com isso ocorre um balanceamento no gasto energético dos enlaces entre todos os satélites, evitando o descarregamento da bateria. Conforme mostrado na Equação (3), como a quantidade de ciclos de recarga/descarga do satélite é limitada, quanto mais descarga, menor é sua vida útil.
No método ERU-DOD ganha-se $54 \%$ a mais de vida útil do satélite na simulação de um dia. Isso significa que se um satélite operasse por 15 anos na proposta de [6], aplicando o método ERU-DOD para a mesma rede de satélites LEO, os satélites operariam por aproximadamente 23 anos. Este resultado representa um ganho relevante, tendo em vista que os satélites possuem custo elevado e suas baterias não podem ser repostas.

O método ERA-DOD gera aproximadamente $10 \%$ a mais de vida útil para as baterias dos satélites na simulação de um dia. Este resultado significa que se o satélite de uma rede LEO operasse por 15 anos na proposta de [6], aplicando o método ERA-DOD para a mesma rede, os satélites operariam por quase 17 anos. Este resultado mostra melhorias significativas pela utilização do método ERA-DOD em redes de satélites LEO.

\subsection{Fontes Bloqueadas}

Na Figura 4 é mostrado o percentual de fontes bloqueadas para os métodos ERU-DOD e ERA-DOD, e para a métrica ECARS. As fontes bloqueadas são os terminais cujas demandas não foram atendidas.

O total de fontes bloqueadas, considerando 1.000 terminais utilizados nas simulações, é calculado pela soma de fontes bloqueadas por falta de energia mínima no satélite mais a soma de fontes bloqueadas por falta de capacidade no enlace.

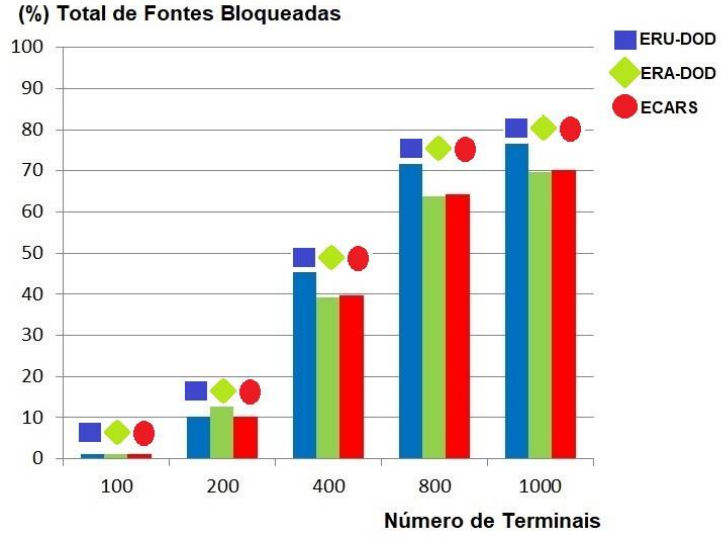

Figura 4 - Total de Fontes Bloqueadas.

Nota-se que a quantidade total de fontes bloqueadas com a utilização do método ERA-DOD e métrica ECARS são quase as mesmas. O método ERA-DOD tem um pouco mais de fontes bloqueadas que os outros dois modelos.

\section{CONCLUSÕES}

Neste artigo foram apresentadas duas propostas de balanceamento de carga no roteamento de pacotes nos satélites de uma constelação LEO, limitando o nível mínimo da bateria, a fim de aumentar a vida útil das baterias dos satélites.

Foram propostos dois métodos, que utilizam a métrica ECARS adaptada e melhorada, em conjunto com o algoritmo de caminho 
com menor custo, Dijkstra, a fim de evitar o roteamento de pacotes por satélites com baixa carga residual de bateria.

O método ERU-DOD desabilita os enlaces cujos satélites não cumprem com o nível mínimo de energia de suas baterias definidas através do limiar $\left(\mathrm{L}_{\mathrm{B}}\right)$. Com isso, ocorre uma diminuição das rotas disponíveis para o tráfego dos satélites, ocasionando um maior número de fontes bloqueadas. Entretanto, evitar a utilização de satélites com pouca energia em suas baterias, ocasiona um ganho de vida útil para as baterias dos satélites.

Por outro lado, o método ERA-DOD penaliza os enlaces cujos satélites que ultrapassaram o limiar mínimo de energia das baterias definidos através do limiar $\left(\mathrm{L}_{\mathrm{B}}\right)$. Mesmo os satélites fora do limiar $\mathrm{L}_{\mathrm{B}}$ podem ser utilizados para o tráfego de dados, gerando menor número de fontes bloqueadas $\mathrm{e}$ melhor balanceamento do tráfego.

Tanto o método ERU-DOD quanto o método ERA-DOD limitam um nível mínimo para a bateria do satélite, tratando de forma distinta e específica os enlaces que não atendem a essa condição. No método ERU-DOD, o enlace é desabilitado. No método ERA-DOD, ao enlace nessa condição é atribuído um peso maior, punindo-o. Dessa forma, ambos os métodos são similares e apenas no roteamento, o simulador toma decisões distintas, não sendo possível um modelo hibrido dos dois métodos.

Os resultados apresentados foram promissores, apresentando ganho de energia residual de $113 \%$ e $29 \%$ para os métodos ERUDOD e ERA-DOD, respectivamente. Além disso, as propostas ERU-DOD e ERA-DOD deste artigo geraram ganhos na vida útil dos satélites de $54 \%$ e $10 \%$, respectivamente.

Os autores de [8] obtiveram um ganho no ciclo de vida de $49,97 \%$ nos seus melhores experimentos, os autores de [9] alcançaram um ganho de $45 \%$, enquanto que o método proposto neste artigo ganha dos demais autores apresentando um aumento no ciclo de vida da bateria de $54 \%$.

Os resultados alcançados com o método ERU-DOD demonstram que o mesmo foi melhor que o método ERA-DOD, quanto à energia residual $(50 \%)$ e em vida útil da bateria (23 anos). Entretanto, os resultados não são satisfatórios em relação a fontes bloqueadas (80\%). No método ERA-DOD, também há um ganho em energia residual $(30 \%)$ e em vida útil da bateria (17 anos), no entanto, esses não são melhores que os apresentados pelo método ERU-DOD. Contudo, quanto às fontes bloqueadas, o método ERA-DOD obtém um valor de $70 \%$, que é melhor do que o obtido pelo método ERU-DOD.

Dessa forma, para priorizar soluções em que se deseja obter melhor desempenho em energia residual e vida útil da bateria, escolhe-se o método ERU-DOD. E, para priorizar soluções em que se deseja obter bom desempenho em vida útil concomitantemente com um melhor percentual de demanda atendida (menor percentual de fontes bloqueadas), utiliza-se o método ERA-DOD.

É importante ressaltar, que este trabalho de pesquisa simula um modelo real da constelação Iridium, com parâmetros usuais, entretanto com ambiente e valores de parâmetros previamente definidos. Apesar do ambiente real utilizar os mesmos parâmetros, seus roteamentos e valores são aleatórios e mutáveis.

A contribuição deste artigo consistiu em aumentar a vida útil das baterias dos satélites, levando em consideração um limiar, a fim de que a bateria do satélite não descarregue por completo. Fazendo o roteamento de pacotes para enlaces que possuem satélites com maior capacidade de bateria.

Em trabalhos futuros, propõe-se expandir os estudos para os seguintes cenários: i) abordar topologia hibrida em redes de satélites, utilizando satélites das diferentes órbitas LEO (Low Earth Orbit - Órbita Terrestre Baixa), MEO (Medium Earth Orbit - Órbita Terrestre Média) e GEO (Geostationary Orbit - Órbita Geoestacionária); ii) incorporar o seguimento terrestre, que consiste nas estações e nos terminais de usuário (VSAT - Very Small Aperture Terminal) e serviço (HUB). Com isso, pretende-se diminuir a quantidade de fontes bloqueadas, melhorando não só o consumo energético e a vida útil do satélite, como também alcançar uma demanda atendida satisfatória.

\section{AGRADECIMENTOS}

Esta pesquisa foi financiada: i) conforme previsto no Art. 48 do decreto $n^{\circ}$ 6.008/2006, pela Samsung Eletrônica da Amazônia Ltda, nos termos da Lei Federal n ${ }^{\circ}$ 8.387/1991, através de convênio $\mathrm{n}^{\circ}$ 004, firmado com o Centro de P\&D em Eletrônica e Tecnologia da Informação da Universidade Federal do Amazonas - CETELI / UFAM; ii) pela coordenação de Aperfeiçoamento de Pessoal de Nível Superior (Capes); iii) pela Fundação de Amparo à Pesquisa do Estado do Amazonas (FAPEAM/ProgramaPPP); iv) fundo setorial de infraestrutura (CT-INFRA); v) MCT/CNPQ; vi) Governo do Estado do Amazonas.

\section{REFERENCIAS}

[1] ITU - International Telecommunication Union. Measuring Digital Development Facts and Figures. Published in Switzerland Geneva, 2019. URL $<$ https://www.itu.int/en/ITUD/Statistics/Documents/facts/FactsFigures2019.pdf >. Acessado em 04 de julho de 2020.

[2] UNESCO - United Nations Educational, Scientific and Cultural Organization, 2019. Assessing Internet Development in Using UNESCO's Internet Universality ROAM-X Indicators BRAZIL. URL $<$ https://cetic.br/media/docs/publicacoes/8/20200117094619/Assessing_Internet _Development_in_Brazil.pdf>. Acessado em 03 de julho de 2020.

[3] Huang, T.; Yang, W.; Wu, J.; Ma, J.; Zhang, X.; and Zhang, D. A Survey on Green 6G Network: Architecture and Technologies. IEEE Access, vol. 7, pp. 175758-175768, 2019

[4] Qu, Z.; Zhang, G.; Cao, H.; and Xie, J. LEO satellite constellation for Internet of Things. IEEE Access, v. 5, p. 18391-18401, 2017.

[5] Su, Y.; Liu, Y.; Zhou, Y; Yuan, J; H. Cao, H.; and Shi, J. Broadband LEO Satellite Communications: Architectures and Key Technologies. IEEE Wireless Communications, vol. 26, no. 2, pp. 55-61, April 2019.

[6] Maceno, F. E. P.; Carvalho, C.; e Mota, E. Roteamento Ciente da Capacidade Consumo Energético em Redes de Satélites LEO. XXXVII SIMPÓSIO BRASILEIRO DE TELECOMUNICAÇÕES E PROCESSAMENTO DE SINAIS, Petrópolis - RJ - Brasil de 29 de setembro a 02 de outubro de 2019.

[7] Hussein, M.; G. Jakllari, G.; and Paillassa, B. Network Pruning for Extending Satellite Service Life in LEO Satellite Constellations. IEEE International Conference on Data Science and Data Intensive Systems, Sydney, NSW, pp. 240-246, 2015. 
[8] Yang, Y.; XU, M.; Wang, D.; and Wang, Y. Towards Energy-Efficient Routing in Satellite Networks. in IEEE Journal on Selected Areas in Communications, vol. 34, no. 12, pp. 3869-3886, Dec. 2016.

[9] Tsuchida, H.; Kawamoto, Y.; Kato, N.; Kaneko, K.; Tani, S.; Uchida, S.; AND Aruga, H. (2020). Efficient Power Control for Satellite-Borne Batteries Using Q-Learning in Low-Earth-Orbit Satellite Constellations. IEEE Wireless Communications Letters, vol. 9, no. 6, pp. 809-812, June 2020

[10] Long, F. Satellite Network Robust QoS-aware Routing. Springer, 2014.

[11] Miniwatts, M. G. Internet Usage Statistics. 2020. URL $<$ https://www.internetworldstats.com/stats.htm>. Acessado em 20 julho 2020.

[12] Chen. C.; and Ekici, E. A Routing Protocol for Hierarchical LEO/MEO Satellite IP Networks. Wireless Netw, vol. 11, pg. 507-521, 2005. 